\title{
Reproducibility, Diversity, and the Crisis of Inference in Psychology
}

\author{
Moin Syed \\ Department of Psychology, University of Minnesota \\ Presented at the Symposium: \\ "Data, Rigor, and Reproducibility in Light of Diversity, Equity, and Inclusion"
}

Friday, 3 December 2021

Organized by the Many Faces of Reproducibility Initiative, University of Minnesota https://reproducibility.umn.edu/

Slides, video, and this paper: https://osf.io/rcyxa/

This is a script of a presentation, and thus does not follow all conventional citations patterns or formalities of scientific writing. It has not been peer reviewed. It could turn into a proper paper someday, however, so I welcome any comments, moin@umn.edu.

This paper is \#22 in the series, "I Got a Lot of Problems with Psychology."

\begin{abstract}
Psychological researchers have long sought to make universal claims about behavior and mental processes. The various crises in psychology-reproducibility, replication, measurement, theory, generalizability-have all demonstrated that such claims are premature, and perhaps impossible using mainstream theoretical and methodological approaches. Both the lack of diversity of samples and simplistic conceptualizations of diversity (e.g., WEIRD, individualism/collectivism) have contributed to an "inference crisis," in which researchers are ill equipped to make sense of group variation in psychological phenomena, particularly with respect to race/ethnicity. This talk will highlight how the lack of sophisticated frameworks for understanding racial/ethnic differences is a major barrier to developing a reproducible, cumulative psychology.
\end{abstract}


I know what you are thinking: not another crisis! Labeling problems in the field as a "crisis" has been en vogue in psychology for ten years now, with the onset of the replication crisis, and has spilled over to most/all other scientific disciplines (Spellman, 2015). Quickly we realized that not only did we have a replication crisis, but a theory crisis (Eronen \& Bringman, 2021), a measurement crisis (Flake \& Fried, 2020), a construct validity crisis (Schimmack, 2019), a generalizability crisis (Yarkoni, 2021), and yes, an inference crisis (Starns et al., 2019). We have debates about whether or not any of these problems are actually crises (Gilbert et al., 2017), as well as sociohistorical analyses of the nature of the "crisis discourse" (Fils, 2019). None of that should distract from the fact that we have serious problems with how we think about and conduct our science.

The purpose of this paper is to interweave some of the various strands of the different crisis domains to begin to form a tapestry that integrates concerns about diversity, reproducibility, generalizability, and scientific rigour. My focus is on psychology, but most of the arguments could generalize to any other discipline. I also primarily focus on diversity with respect to race/ethnicity, but also draw on some research on sex/gender, but again, most of the arguments generalize to any dimension of diversity. As usual, I am long on criticism and short on solutions, in part because I want to urge us to think more deeply-and differently-about the nature of the problems so that we can generate useful answers, and not rush to solutions for solutions' sake.

Before diving in, it is important to get straight on terms and definitions. Unfortunately-and ironically given the focus of reforms - frequently used terms are not defined or understood in consistent ways. I endorse The Turing Way Community's (2021) framework that separates out whether a given project is using the same or different data from whether it relies on the same or different analysis.

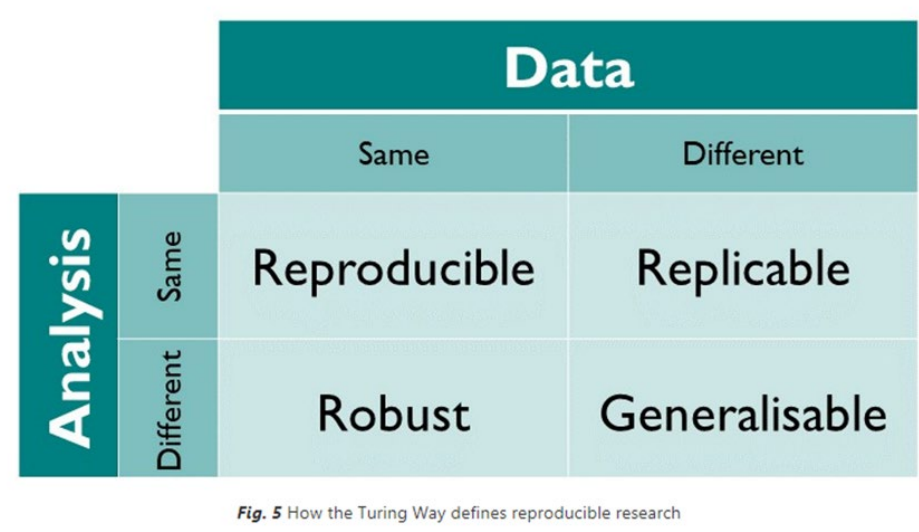

This framework makes a useful distinction between reproducibility, which is based on the same analysis of the same data-essentially, if someone ran the same analysis scripts with the same data, would they get the same results? - and replicability, which relies on the same analysis strategy but with different data (i.e., conducting the "exact" same study with a new sample). 
These two terms are commonly used interchangeably, a practice that was not helped at all by the Open Science Collaboration's landmark 2015 article, "Estimating the reproducibility of psychological science," which was about replicability, not reproducibility. Anyway, The Turing Way's framework contains two other cells: robustness is using different analyses with the same data, examining whether the findings hold up across different modeling specifications, and generalizability uses both different analyses and different data, and thus speaks to the boundary conditions of the observed findings.

Diversity has largely been seen as a problem of generalizability. It has been long known and documented that psychology has relied on a thin slice of humanity in generating its knowledge-primarily the White, American/Western European middle class-yet it treats that knowledge as universal and true for all of humanity (Arnett, 2008; Graham, 1992; Guthrie, 1976; Hartmann et al., 2013; Henrich et al., 2010; Roberts et al., 2020). The quest for universals can be traced back through the history of empirical psychology and the field's persistent physics envy that has both downplayed cultural/contextual factors and valorized quantitative methods (Syed, 2021a; Syed \& McLean, 2021). As part of the open science movement, however, there have been increasing attempts to move away from this kind of thinking and attempt to build a more contextualized field. This is being done by expanding the global range of samples and researchers, as exemplified by the Psychological Science Accelerator (Moshontz et al., 2018) and the "Many" projects: Many Labs (Klein et al., 2018), Many Babies (Frank et al., 2017), and \#EEGMany Labs (Pavlov et al., 2021), to name a few. These are valuable initiatives that have already yielded impressive results, but they also primarily conceptualize diversity and generalizability as procedural problems to be solved; if we include more researchers from around the world, and draw samples from more regions, then we will have solved the problems of the lack of diversity and generalizability.

However, another set of solutions to the problem of generalizability is to more accurately represent the reach of our empirical work, to whom and what conditions it should actually generalize. These solutions can be broadly thought of as emphasizing "calibrated claims" (Vazire, 2018), and have been supported through arguments from researchers (Simons et al., 2017; Yarkoni, 2021) and policies enacted at journals (Arnett, 2005; SRCD. 2020). Rather than primarily a procedural problem, this set of solutions identifies the issues surrounding diversity and generalizability as an inferential problem, a problem of how we draw conclusions from our data. Moreover, addressing the procedural problem - that is, diversifying samples and researchers-still leaves us dealing with the inferential problem of needing to appropriately calibrate claims, suggesting that this is a central barrier to the advancement of our science ${ }^{1}$.

Of course, the inferential problems plaguing psychology have been long discussed (e.g., Meehl, 1967), but to my knowledge the first use of the term "inferential crisis" was in Starns et al. (2019). They defined the inferential crisis as, "a surprisingly high likelihood of drawing incorrect theoretical conclusions even if [researchers] are working with reliable, replicable data

\footnotetext{
${ }^{1}$ In many ways, all that I have argued here is wrong, and what we really have is a structural problem, which is what has led to both underrepresentation of different populations and the barriers to inference.
} 
patterns," (Starns et al., 2019, p. 336). The problem of how we draw conclusions from our data seems to be the central issue facing psychological research. Whether we are talking about problems of replication, measurement, or theory, in all cases what we are highlighting is that our inferences are compromised through the institutionalized use of poor practices.

Accordingly, rather than arguing about what the "true" crisis is-which is more important and in need of immediate attention-perhaps the inferential crises can serve as the central, overarching problem that both subsumes all of the other crises and highlights the ways in which they are interconnected (see also Crüwell, 2021, for a similar argument).

With this set up in mind, I now come to my central point: that problems of inference are a major barrier to successfully diversifying psychological science. The default model in psychological research is universalism, in which any individual observation is seen as a replaceable representative of all of humanity (Figure 2, left). There are, of course some qualifiers here. Because we operate in a structurally imbalanced system, where power is not equally distributed among different groups of scientists, this is actually only true for the dominant White North American and Western European psychology. Individuals who fall outside of the dominant groups are required to identify and justify their existence; they are not replaceable members of humanity. That said, my attention at the moment is to the mainstream of the field, which very much follows this "replaceable humanness" model.
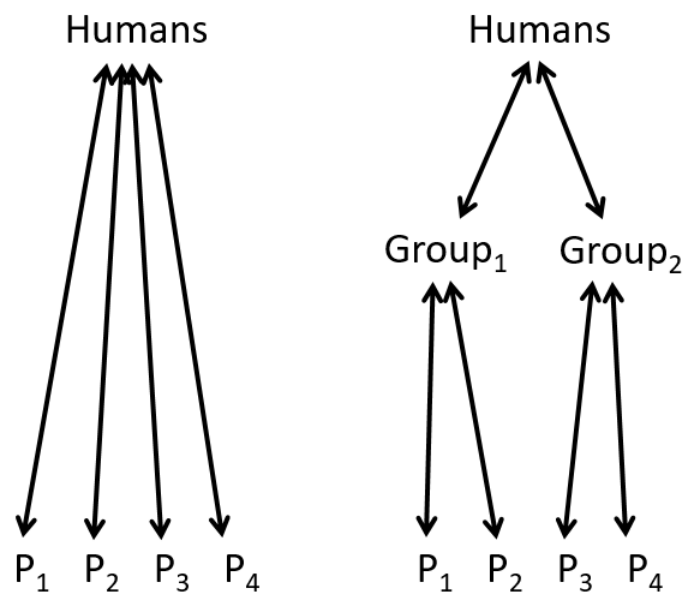

Figure 2. Two models of psychological inference

One of the challenges to attending to diversity in the field is that it adds an additional, intermediate layer to the model (Figure 2, right). Rather than individuals simply being part of the broad category of humans, individuals are members of different groups, which are in turn part of the broader set of humans (Syed, 2020). Adding just this one intermediate layer leads to a much higher level of complexity in terms of what any psychological theory must account for. We are relatively well-equipped to understand the individual variation within groups, as this is just a smaller-scale version of the individual-human model. That said, we ironically tend to do a relatively poor job of attending to these variations, especially when comparing groups. 
In contrast, I argue that we are ill-equipped to understand the similarities and differences between and among groups. We can measure differences between groups, but appropriately interpreting those differences is another matter. Importantly, groups themselves do not have any explanatory value. There is no psychological meaning inherent to groups themselves, and groups do not have any causal impact on psychological variability. Rather, groups are used as proxies to represent a large bundle of specific constructs that vary within those groups (e.g., discrimination, identity, socialization, values, stereotypes, attitudes; see Helms et al., 2005, Gjerde, 2004), many of which are unknown or poorly measured.

Thus, there is an inferential chasm between groups and data/observations. This is a major problem due to the lack of rigorous theory, very poor measurement, scant attention to construct validity, over-privileging explanation vs. description, and little emphasis on replication. Inattention to all of these issues together constitutes the inferential crises, in psychology in general, but specifically in the context of understanding similarities and differences between groups.

Moreover, the inferential chasm is not simply left to exist on its own. Rather, the wide-open spaces must be filled in by researchers. Because we lack solid theories to explain group differences, researchers are left to fill the space with their own preferred inferential account. Observed group variation could be due to environmental, structural, genetic, and/or evolutionary factors - or, of course, they could just be noise. It is rare that a study design actually adjudicates among these different putative explanations, and again, there is rarely a rigorous theoretical account that could be used as a possible explanation. Thus, what we have is essentially a projective inferential space, where inferences about group explanation simply reflect the researchers preexisting beliefs about the nature of those differences.

A classic example of this problem is the highly controversial and politically-charged research on racial differences in standardized testing. The cause of the differences has not been reliably identified empirically, but that has not stopped researchers from interpreting the differences as structural, environmental, or genetic, depending on their preferred view (Helms et al., 2005). Another interesting case is BiDil (Isosorbide dinitrate/hydralazine), the first and only racespecific medication approved by the U.S. Food and Drug Administration (see Brody \& Hunt, 2006). BiDil is approved to treat heart disease only among Black Americans. I find this case particularly interesting because it seems to reinforce the fallacious idea that there is a biological basis for race; if not, why would there be medication specifically for use among only one racial group? Remarkably, there have been not been any studies that have identified a biological or genetic rationale for the medication. The approval was based on an initial study that included a post-hoc interaction between White and Black participants that was of questionable evidentiary value, followed by a trial that enrolled only Black participants showing that it was effective at reducing mortality. The evidence that the medication should be race-specific is extremely weak. Given the evidence, literally any possible explanation could be used to make sense of the findings, and so we have an inferential chasm that will be filled by researchers' own preconceived beliefs, stereotypes, and priorities. 
Now, to be clear, I am not saying that we do not have any inferential framework for understanding group variation. I am arguing that they are insufficient; or more bluntly, that they are not very good.

One of the most widely used frameworks for understanding global group variation is Hofstede's (1980) individualism/collectivism model of cultural syndromes (or national traits). This binary model suggest that countries are either individualistic, in that they promote individuality, autonomy, and personal agency, or they are collectivistic, emphasizing relationships, connections, and hierarchies. Markus and Kitayama (1991) famously extended the model beyond cultural syndromes to individual selves, arguing that individualistic cultures facilitate the development of independent self-construals, whereas collectivistic cultures facilitate interdependent self-construals. These self-construals, in turn, have a profound impact on nearly any aspect of human psychological variation. Individualism/collectivism and independence/interdependence are wildly popular across the social sciences, and seemingly serve as the de facto explanation for any observed cultural variation in psychological phenomena. The problems, however, are many. Whether specific countries are individualistic or collectivistic is a debated question (Takano \& Osaka, 1999), in part because of the fact that no country is either one or the other. Both cultural traits/values exist in all cultures, and their relevance for any specific observation will vary based on a whole host of contextual factors (Tamis-LeMonda et al., 2005). Additionally, the original approach to indexing cultures as individualistic or collectivistic suffered from major limitations of sampling, measurement, and inference (see McSweeney, 2002). Most germane to the current argument, the actual empirical studies bear little resemblance to the underlying model. As Matsumoto (1999) pointed out many years ago (and is still true today), culture is rarely measured, and self-construal is rarely measured. Rather, the vast majority of studies in this framework simply take samples from different countries and compare them on some psychological construct. With this kind of design, literally any explanation for observed similarities and differences is possible, and so we have an inferential chasm that will be filled by researchers' own preconceived beliefs, stereotypes, and priorities.

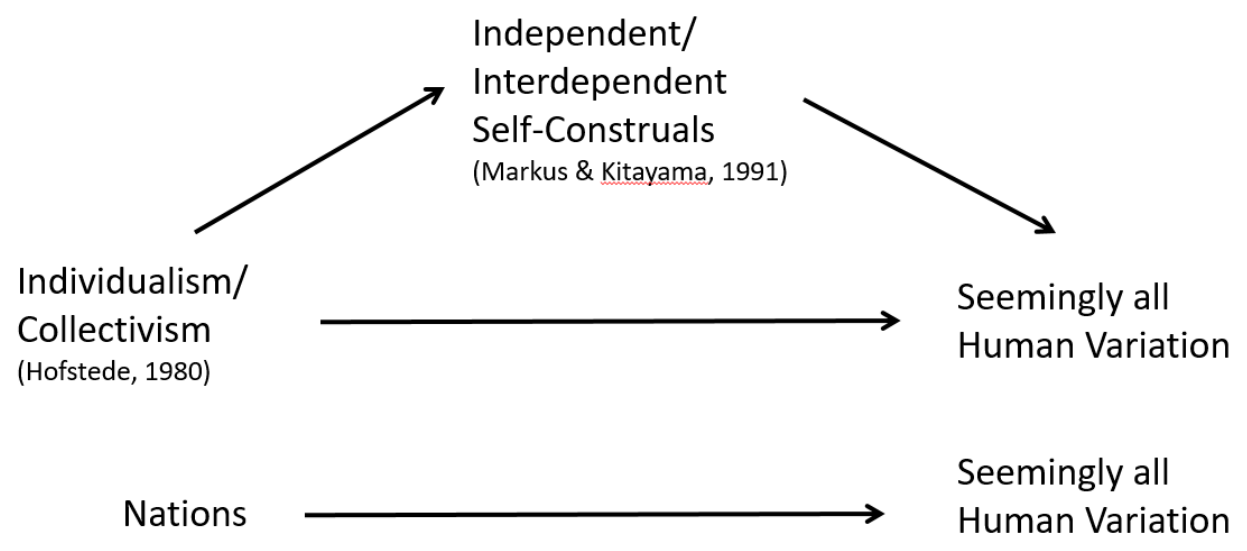

Figure 3. This disconnect between theory (top) and data (bottom) in research on cultural syndromes. Adapted from Matsumoto (1999). 
More recently, Henrich et al. (2010) proposed WEIRD as a framework for understanding global variation, arguing that much of the research in behavioral sciences is based on samples that come from societies that are Western, Educated, Industrialized, Rich, and Democratic. This, too, has been a wildly popular framework for conceptualizing group variation, but its inferential value is extremely low. Despite its popularity, there have been a number of critiques levied at it, including that it does not engage at all with race/ethnicity (Clancy \& Davis, 2019), that it creates a problematic dichotomy between WEIRD and non-WEIRD societies (Ghai, 2021), that it is an acronym-driven concept (Syed, 2021b), and that the acronym distracts from the more important, nuanced points that underlie it (Dutra, 2021). Once again, though, to my central argument, the major problem is the lack of precision and evidence associated with it, that it leaves an inferential chasm that will be filled by researchers' own preconceived beliefs, stereotypes, and priorities.

These are just two examples, but they are also without a doubt the two most often cited and discussed frameworks for understanding global group variation. But the problem is not limited to that context of group formulation. Taking a detour to research on sex/gender differences highlights the many problematic methodological practices that feed into the inferential crisis.

A recent paper by Garcia-Sifuentes and Maney (2021) contained a review of methodological practices around sex differences in the biological sciences, and the resulting picture was not good. Among many other findings, they observed that sex is frequently included as a factor in a factorial design, but that more often than not, researchers do not actually test for the statistical interaction of sex in their models. This, on its own, is not a problem, as researchers may not have hypotheses or exploratory goals that requires such a test. They also found, however, that nearly $70 \%$ of studies that claimed the presence of a sex-specific effect did not actually test for interactions (about 35\% that claimed the absence of an effect also did not test the interaction). Rather, sex-specific effects were frequently examined through separate models, comparing a significant $p$-value in one group to a non-significant $p$-value in another group. As the saying goes, the difference in statistical significance does not imply a statistically significant difference. This work was focused on the biological sciences, but I can assure you (as could anyone who has paid any attention at all) the same practices can be seen in psychological research. The inferential chasm is thus being filled in with some shaky empirical claims, made sense via researchers' own preconceived beliefs, stereotypes, and priorities

Indeed, Persson and Pownall (2021) recently argued that testing for sex differences can be thought of as a form of $p$-hacking, or serial testing of alternative models until one "turns up" statistically significant. They argue, "sex differences can in theory be analyzed with any given neuroscientific dataset and may therefore be a convenient alternative analysis when other core hypotheses within the data are not confirmed" (p. 496). In other words, the motivation for testing for sex differences may not be a priori theoretical arguments for the presence of sex differences, but rather they need to salvage the publishability of a study under a system of intense publication bias. Similarly, we have argued there exists a "burden of the diverse sample" (Syed \& Kathawalla, 2021). If authors are clear about the demographic diversity of the sample, be it sex/gender, race/ethnicity, social class, or any other form of demographic 
variation, there is often press from editors and reviewers to test for differences along those dimensions. This pressure is seemingly sensible in the name of robustness and generalizability, but absent any theoretical understanding of what differences might be observed, and more importantly what they may mean, any observed differences may be spurious and lend themselves to ad-hoc explanations. Once again, the inferential chasm is being filled in with some shaky empirical claims-or at least not intentional ones-made sense via researchers' own preconceived beliefs, stereotypes, and priorities.

To make matters worse, groups themselves are frequently ill-defined. Taking a look at the U.S. Census categories over time highlights the socially-constructed nature of race/ethnicity. It was not until 2000 that respondents could choose more than one category, but obviously people have had mixed-ethnic/racial backgrounds all along; we just did not attend to them. The subjectivity and constructed nature of racial/ethnic categories becomes even more apparent when looking internationally, where even in supposedly "culturally-similar" Western Europe we do not seen racial/ethnic categories that bear any resemblance to what is used in the U.S. (Gyberg et al., 2021; Juang et al., 2021; Jugert et al., 2021). Similar problems come up with other group categories: what countries are Western? who is middle-class? how do you define gender? sexuality? immigrant status? And so on.

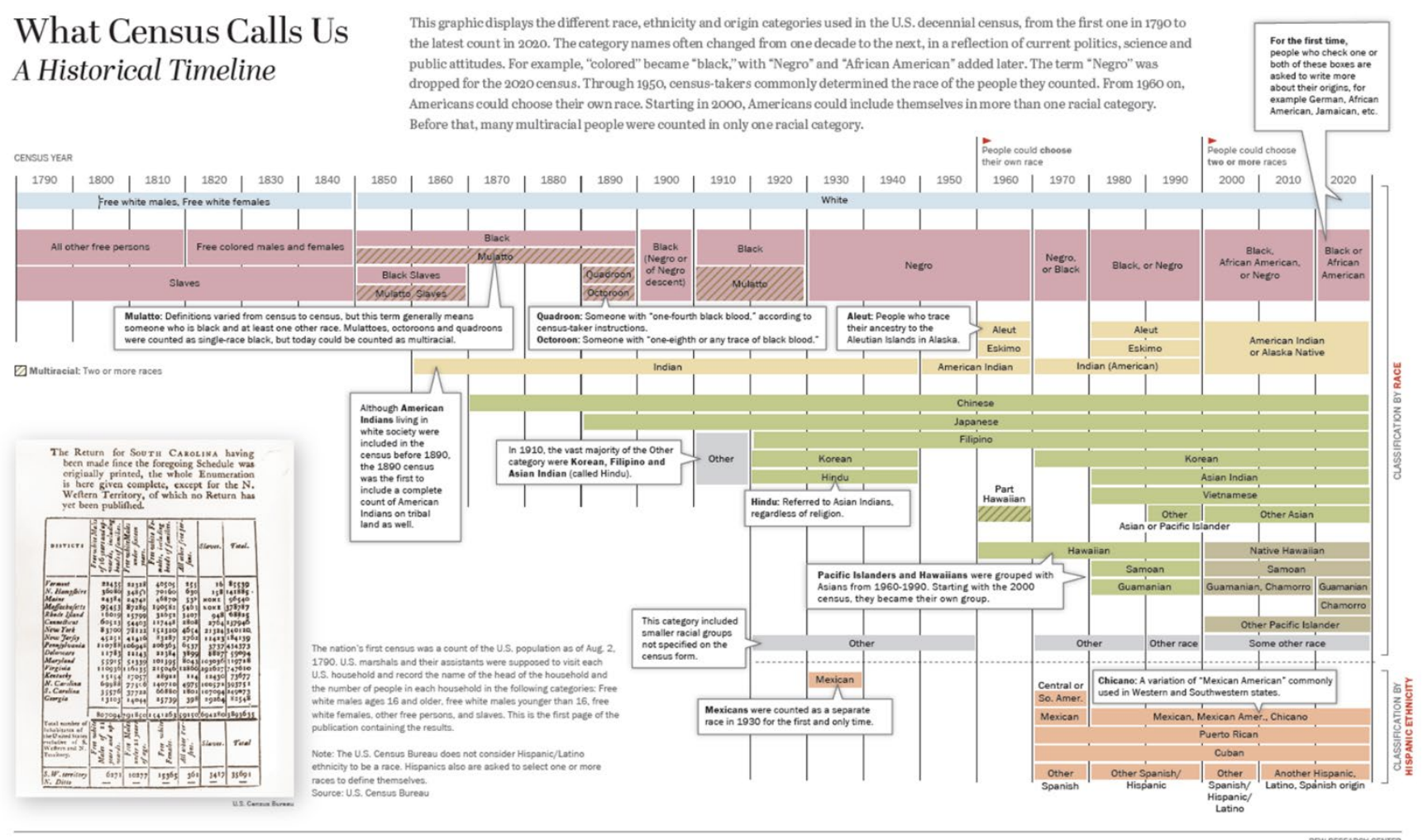

Figure 4. The U.S. Census racial categories over time. From https://www.pewresearch.org/interactives/whatcensus-calls-us/ 
Taking this all together, we can measure variations across groups, but we have weak or nonexistent theories to help explain and predict group differences, and those groups themselves are not clearly, consistently, or accurately defined. Thus, we have a vast inferential chasm, and in light of the calls to diversify our science, we must acknowledge that we are ill-equipped to understand that diversity in substantively meaningful ways.

But wait, it gets worse.

Coming back full circle to The Turing Way's framework for understanding reproducibility, we can see quite clearly that researchers do not play nicely with respect to these different quadrants, shifting between them as necessary to protect their preferred theories. A particularly pernicious example of this is the slippage between replication and generalizability. During the massive attention to replications in psychology during the mid-2010s, original researchers of studies that failed subsequent replications would argue that such results were not failures to replicate at all, but rather were data points that were informative of the boundary conditions of the results (i.e., the result was about generalizability, not replication). Taking this argument even further, Van Bavel et al. (2016) argued that the reason for the lower rate of replication in social psychology relative to cognitive psychology is that the former is more "contextually sensitive," and thus should not be expected to replicate under just any conditions. This argument, on its face, is quite reasonable, but in reality it is not reasonable at all. This set of explanations is always post-hoc, after the failures to replicate are known, and the original papers do not mention contextual sensitivity of the findings at all, but rather put forward universal, invariant claims. Accordingly, this strategy is a prime example of what Imre Lakatos described as a degenerative line of research, where arguments are made to defend a theory to keep it alive, rather than making progressive modifications in light of new evidence.

A less frequently discussed form of slippage within The Turing Way framework is between robustness and replication. For example, testing for sex differences in a sample, outside of having specific hypotheses, is most appropriately conceptualized as robustness testing; do the results hold up for different groups, different models, etc. However, if the results are not robust across groups, and the target finding only holds for a specific group, that can be taken by others as evidence for replication, even though the group-specific nature of the finding had not been previously specified.

Finally, to not leave reproducibility out of the discussion, we also see degenerative defenses when there are problem there. When readers attempt to reproduce a published article's results, and find differences between what is observed and reported in the article, it is common for the correction notice to state that the corrections "did not change the conclusions of the study," even when those corrections are substantial. Seemingly nothing can alter the conclusions of a study once published.

As I said at the outset, my treatment of this issue would be long on criticism and short on solutions, but I will end with one solution, the solution I always point to when discussing these issues: we need to place greater value on observation and description. As a discipline we are 
hyper-focused on developing explanations-identifying mechanisms, generating and testing theories, etc. That all makes sense, as in many ways that is where the most potentially "exciting" and "sciencey" work happens. However, our obsession with identifying explanations is a manifestation of our physics envy, which has led us to prematurely emphasize explanation without having spent sufficient time and attention to observation and description, which are the necessary foundations to any adequate explanatory account (Scheel et al., 2021; Syed \& McLean, 2021). How can we explain phenomena that we do not fully understand? If we spent more time on these foundational steps, then we would be able to build up theories and explanations for group variations, and perhaps have a proper science of psychological diversity. It is not too late to shift our attention, but we must have the will to do so.

\section{References}

Arnett, J. J. (2005). The Vitality Criterion: A new standard of publication for Journal of Adolescent Research. Journal of Adolescent Research, 20(1), 3-7.

Arnett, J. J. (2008). The neglected 95\%: Why American psychology needs to become less American. American Psychologist, 63(7), 602-614. https://doi.org/10.1037/0003$\underline{066 X .63 .7 .602}$

Brody, H., \& Hunt, L. M. (2006). BiDil: assessing a race-based pharmaceutical. The Annals of Family Medicine, 4(6), 556-560.

Clancy, K. B. H., \& Davis, J. L. (2019). Soylent Is People, and WEIRD Is White: Biological Anthropology, Whiteness, and the Limits of the WEIRD. Annual Review of Anthropology, 48(1), 169-186. https://doi.org/10.1146/annurev-anthro-102218-011133

Crüwell, S. (2021). Reframing the replication crisis as a crisis of inference. Manuscript in preparation.

Dutra, N. B. (2021). Commentary on Apicella, Norenzayan, and Henrich (2020): Who is going to run the global laboratory of the future? Evolution and Human Behavior, 42(3), 271-273. https://doi.org/10.1016/i.evolhumbehav.2021.04.003

Eronen, M. I., \& Bringmann, L. F. (2021). The Theory Crisis in Psychology: How to Move Forward. Perspectives on Psychological Science, 1745691620970586. https://doi.org/10.1177/1745691620970586

Flis, I. (2019). Psychologists psychologizing scientific psychology: An epistemological reading of the replication crisis. Theory \& Psychology, 29(2), 158-181.

Flake, J. K., \& Fried, E. I. (2020). Measurement schmeasurement: Questionable measurement practices and how to avoid them. Advances in Methods and Practices in Psychological Science, 3(4), 456-465. https://doi.org/10.1177\%2F2515245920952393

Frank, M. C., Bergelson, E., Bergmann, C., Cristia, A., Floccia, C.,Gervain, J., ... \& Lew-Williams, C. (2017). A collaborative approach to infant research: Promoting reproducibility, best practices, and theory-building. Infancy, 22(4), 421-435. https://doi.org/10.31234/osf.io/27b43

Garcia-Sifuentes, Y., \& Maney, D. L. (2021). Reporting and misreporting of sex differences in the biological sciences. ELife, 10, e70817. https://doi.org/10.7554/eLife.70817 
Ghai, S. (2021). It's time to reimagine sample diversity and retire the WEIRD dichotomy. Nature Human Behaviour, 1-2. https://doi.org/10.1038/s41562-021-01175-9

Gilbert, D. T., King, G., Pettigrew, S., \& Wilson, T. D. (2016). Comment on “Estimating the reproducibility of psychological science". Science, 351(6277), 1037-1037.

Gjerde, P. F. (2004). Culture, Power, and Experience: Toward a Person-Centered Cultural Psychology. Human Development, 47(3), 138-157. https://doi.org/10.1159/000077987

Graham, S. (1992). "Most of the subjects were White and middle class": Trends in published research on African Americans in selected APA journals, 1970-1989. American Psychologist, 47(5), 629. https://doi.org/10.1037/0003-066X.47.5.629

Guthrie, R. V. (1976). Even the rat was white: A historical view of psychology. Allyn \& Bacon.

Gyberg, F., Svensson, Y., Wängqvist, M., \& Syed, M. (2021). Discrimination and its relation to psychosocial well-being among diverse youth in Sweden. New Directions for Child and Adolescent Development, 176, 163-181. 10.1002/cad.20399

Hartmann, W. E., Kim, E. S., Kim, J. H. J., Nguyen, T. U., Wendt, D. C., Nagata, D. K., \& Gone, J. P. (2013). In Search of Cultural Diversity, Revisited: Recent Publication Trends in CrossCultural and Ethnic Minority Psychology. Review of General Psychology, 17(3), 243-254. https://doi.org/10.1037/a0032260

Helms, J. E., Jernigan, M., \& Mascher, J. (2005). The meaning of race in psychology and how to change it: A methodological perspective. American Psychologist, 60(1), 27-36. https://doi.org/10.1037/0003-066X.60.1.27

Henrich, J., Heine, S. J., \& Norenzayan, A. (2010). The weirdest people in the world? Behavioral and Brain Sciences, 33(2-3), 61-83. https://doi.org/10.1017/S0140525X0999152X

Hofstede, G. (1980). Culture's consequences: International differences in work-related values. Beverly Hills, CA: Sage.

Juang, L. P., Moffitt, U., Schachner, M. K., \& Pevec, S. (2021). Understanding Ethnic-Racial Identity in a Context Where "Race" Is Taboo. Identity, 1-15. https://doi.org/10.1080/15283488.2021.1932901

Jugert, P., Kaiser, M. J., Ialuna, F., \& Civitillo, S. (2021). Researching race-ethnicity in race-mute Europe. Infant and Child Development, e2260.

Klein, R. A., Vianello, M., Hasselman, F., Adams, B. G., Adams, R. B., Alper, S., Aveyard, M., Axt, J. R., Babalola, M. T., Bahník, Š., Batra, R., Berkics, M., Bernstein, M. J., Berry, D. R., Bialobrzeska, O., Binan, E. D., Bocian, K., Brandt, M. J., Busching, R., ... Nosek, B. A. (2018). Many Labs 2: Investigating Variation in Replicability Across Samples and Settings. Advances in Methods and Practices in Psychological Science, 1(4), 443-490. https://doi.org/10.1177/2515245918810225

Markus, H. R., \& Kitayama, S. (1991). Culture and the self: Implications for cognition, emotion, and motivation. Psychological Review, 98(2), 224. https://doi.org/10.1037/0033295X.98.2.224

Matsumoto, D. (1999). Culture and self: An empirical assessment of Markus and Kitayama's theory of independent and interdependent self-construals. Asian Journal of Social Psychology, 2(3), 289-310. https://doi.org/10.1111/1467-839X.00042

McSweeney, B. (2002). Hofstede's Model of National Cultural Differences and their Consequences: A Triumph of Faith - a Failure of Analysis. Human Relations, 55(1), 89118. https://doi.org/10.1177/0018726702551004 
Meehl, P. E. (1967). Theory-testing in psychology and physics: A methodological paradox. Philosophy of Science, 34(2), 103-115.

Moshontz, H., Campbell, L., Ebersole, C. R., IJzerman, H., Urry, H. L., Forscher, P. S., Grahe, J. E., McCarthy, R. J., Musser, E. D., Antfolk, J., Castille, C. M., Evans, T. R., Fiedler, S., Flake, J. K., Forero, D. A., Janssen, S. M. J., Keene, J. R., Protzko, J., Aczel, B., ... Chartier, C. R. (2018). The Psychological Science Accelerator: Advancing Psychology Through a Distributed Collaborative Network. Advances in Methods and Practices in Psychological Science, 1(4), 501-515. https://doi.org/10.1177/2515245918797607

Open Science Collaboration. (2015). Estimating the reproducibility of psychological science. Science, 349(6251).

Pavlov, Y. G., Adamian, N., Appelhoff, S., Arvaneh, M., Benwell, C. S., Beste, C., ... \& Mushtaq, F. (2021). \# EEGManyLabs: Investigating the replicability of influential EEG experiments. Cortex.

Persson, S., \& Pownall, M. (2021). Can Open Science be a Tool to Dismantle Claims of Hardwired Brain Sex Differences? Opportunities and Challenges for Feminist Researchers.

Psychology of Women Quarterly, 45(4), 493-504. https://doi.org/10.1177/03616843211037613

Roberts, S. O., Bareket-Shavit, C., Dollins, F. A., Goldie, P. D., \& Mortenson, E. (2020). Racial Inequality in Psychological Research: Trends of the Past and Recommendations for the Future. Perspectives on Psychological Science, 15(6), 1295-1309. https://doi.org/10.1177/1745691620927709

Scheel, A. M., Tiokhin, L., Isager, P. M., \& Lakens, D. (2021). Why Hypothesis Testers Should Spend Less Time Testing Hypotheses. Perspectives on Psychological Science, 16(4), 744755. https://doi.org/10.1177/1745691620966795

Schimmack, U. (2019). The Validation Crisis in Psychology. PsyArXiv. https://psyarxiv.com/q247m/

Simons, D. J., Shoda, Y., \& Lindsay, D. S. (n.d.). Constraints on Generality (COG): A Proposed Addition to All Empirical Papers. 6.

Spellman, B. A. (2015). A Short (Personal) Future History of Revolution 2.0. Perspectives on Psychological Science, 10(6), 886-899. https://doi.org/10.1177/1745691615609918

SRCD (2020). New Sociocultural Policy Enacted Across all SRCD Journals. https://www.srcd.org/news/new-sociocultural-policy-enacted-across-all-srcd-journals

Starns, J. J., Cataldo, A. M., Rotello, C. M., Annis, J., Aschenbrenner, A., Bröder, A., Cox, G., Criss, A., Curl, R. A., Dobbins, I. G., Dunn, J., Enam, T., Evans, N. J., Farrell, S., Fraundorf, S. H., Gronlund, S. D., Heathcote, A., Heck, D. W., Hicks, J. L., ... Wilson, J. (2019). Assessing Theoretical Conclusions With Blinded Inference to Investigate a Potential Inference Crisis. Advances in Methods and Practices in Psychological Science, 2(4), 335-349. https://doi.org/10.1177/2515245919869583

Syed, M., \& Kathawalla, U. K. (2021). Cultural psychology, diversity, and representation in open science. In K. C. McLean (Ed.), Cultural methods in psychology: Describing and transforming cultures (pp. 427-454). New York: Oxford University Press.

Syed, M., \& McLean, K. C. (2021). Disentangling paradigm and method can help bring qualitative research to post-positivist psychology and address the generalizability crisis. Behavioral and Brain Sciences. 
Syed, M. (2020). Whither the "White control group"? On the benefits of a comparative ethnic minority psychology. PsyArXiv. 10.31234/osf.io/n4p73

Syed, M. (2021). It's 2 x 2 designs all the way down: Social psychology's over-reliance on experiments needlessly restricts diversity in the field. PsyArXiv. 10.31234/osf.io/u89e2

Syed, M. (2021). WEIRD Times: Three Reasons to Stop Using a Silly Acronym. Get Syeducated. https://getsyeducated.blogspot.com/2021/06/weird-times-three-reasons-to-stopusing.html

Takano, Y., \& Osaka, E. (1999). An unsupported common view: Comparing Japan and the US on individualism/collectivism. Asian Journal of Social Psychology, 2(3), 311-341.

Tamis-LeMonda, C. S., Way, N., Hughes, D., Yoshikawa, H., Kalman, R. K., \& Niwa, E. Y. (2007). Parents' Goals for Children: The Dynamic Coexistence of Individualism and Collectivism in Cultures and Individuals. Social Development, 0(0), 071124114012002-??? https://doi.org/10.1111/j.1467-9507.2007.00419.x

The Turing Way Community. (2021). The Turing Way: A handbook for reproducible, ethical and collaborative research (1.0.1). Zenodo. https://doi.org/10.5281/zenodo.5671094

Vazire, S. (2018). Implications of the Credibility Revolution for Productivity, Creativity, and Progress. Perspectives on Psychological Science, 13(4), 411-417. https://doi.org/10.1177/1745691617751884

Yarkoni, T. (2021). The Generalizability Crisis. Behavioral and Brain Sciences. 